Investigating individual driver performance - applying DEA on simulator data Peer-reviewed author version

BABAEE, Seddigheh; SHEN, Yongjun; HERMANS, Elke; WETS, Geert \& BRIJS, Tom (2014) Investigating individual driver performance - applying DEA on simulator data. In: Wen Z., Lee T. (Ed.). PRACTICAL APPLICATIONS OF INTELLIGENT SYSTEMS, ISKE 2013, SPRINGER-VERLAG BERLIN, p. 623-635.

DOI: $10.1007 / 978-3-642-54927-4 \_59$

Handle: http://hdl.handle.net/1942/16219 


\title{
Investigating individual driver performance - applying DEA on simulator data
}

\author{
Seddigheh Babaee, Yongjun Shen, Elke Hermans, Geert Wets, Tom Brijs \\ Transportation Research Institute (IMOB), Hasselt University, Wetenschapspark 5 bus 6, 3590 \\ Diepenbeek, Belgium \\ Phone: +3211269164 Fax: +3211269199 \\ E-mail: \{seddigheh.babaee, yongjun.shen, elke.hermans, geert.wets, tom.brijs $\} @$ uhasselt.be
}

\begin{abstract}
The main purpose of the present study is to investigate individual driver's behavior by using the data from a driving simulator, in order to distinguish the best drivers and identify the problematic behavior of 'underperforming' drivers. To this end, 129 participants with different age and gender were enrolled to take part in a particular simulator scenario (i.e., curve taking) and their speed, acceleration and lateral position, the three most important driving performance indicators based on literature review, were monitored at various points (before, during and after the curve) while driving a STISIM simulator. As a widely accepted tool for performance monitoring, benchmarking and policy analysis, the concept of composite indicators (CIs), i.e., combining single indicators into one index score, was employed, and the technique of data envelopment analysis - an optimization model for measuring the relative performance of a set of decision making units, or drivers in this study was used for the index construction. Based on the results from the model, all drivers were ranked, and valuable insight were gained by comparing the relative performance of each driver. Finally, the sensitivity of the results was examined.
\end{abstract}

Keywords: Data Envelopment Analysis, Composite indicators, Driver`s relative performance, Driving simulator.

\section{Introduction}

Road safety continues to be one of the world's most serious public health issues. Although road safety actions taken so far have been effective, the number of road fatalities and casualties remain unacceptably high. Every year, the lives of almost 1.24 million people are cut short as a result of road traffic crashes, and between 20 to 50 million more people suffer non-fatal injuries, with many incurring a disability as a result of their injury [WHO, 2013]. Accordingly, the United Nations has declared the period 2011-2020 as the "Decade of Action for Road Safety". The aim is to stabilize and then to reduce mortality caused by road crashes worldwide (http://www.who.int/roadsafety/decade_of_action/plan/en/).

To make progress on road safety, it is widely agreed that rather than only focusing on crash data, more policy attention should be paid to the underlying risk factors influencing safety, which can be classified as related to human, vehicle, road and environment. As mentioned in a large number of in-depth accident investigation studies, inappropriate road user behavior is the major contributory factor in over $90 \%$ and the sole cause in $57 \%$ of all crashes [Treat et al., 1977, Rumer, 1982, Green and Senders, 2004]. Moreover, following from the 
modal split (and the majority of kilometers travelled by drivers), drivers represent a large share in the number of road fatalities (See Table 1). As a result, better understanding the behavior of different drivers is an essential component for future safety improvements on the roadways of the world.

Table 1. Share of road fatalities by road user type and road category [European road statistic, 2012]

\begin{tabular}{|l|l|l|l|l|}
\hline Road user type & Rural & Motorway & Urban & Total \\
\hline Driver & $69.10 \%$ & $61.07 \%$ & $50.47 \%$ & $61.31 \%$ \\
\hline Pedestrian & $10.68 \%$ & $10.65 \%$ & $36.70 \%$ & $20.78 \%$ \\
\hline Passenger & $20.16 \%$ & $28.22 \%$ & $12.81 \%$ & $17.87 \%$ \\
\hline Unkown & $0.05 \%$ & $0.05 \%$ & $0.02 \%$ & $0.04 \%$ \\
\hline
\end{tabular}

Over the last decades, although numerous studies have been carried out with the purpose of investigating driver behavior on safety, there is limited research on individual driver risk in the traffic and human factor engineering field. This is an important gap because identifying and analyzing high-risk drivers will greatly benefit the development of proactive driver education programs and safety countermeasures. Therefore, the aim of this study is to evaluate relative performance of (car) drivers at the individual level, using data from a fixedbased driving simulator. The technique of data envelopment analysis (DEA) in general, and the multiple layer DEA based composite index model in particular, are employed which to our knowledge is the first time that DEA is used for the evaluation of drivers' performance. The results will lead to distinguish the best drivers and advise some improvements to the underperforming drivers.

\section{Methodology}

Data Envelopment Analysis (DEA), originally developed by Charnes, Cooper, and Rhodes (CCR) in 1978, is a non-parametric optimization technique that uses linear programming to measure the relative efficiency of a set of decision making units (DMUs), or drivers in this study. It has become an alternative and a complement to traditional central-tendency analyses and has a number of advantages. As Golany and Roll (1989) pointed out, DEA can be applied to: rank the DMUs, evaluate the effectiveness of programs or policies, identify sources of inefficiency, and create a quantitative basis for reallocating.

In recent years, there has been an increasing interest in the application of DEA for composite indicator (CI) construction, in which single indicators are aggregated into one index score that provides comparisons of DMUs in complex and sometimes elusive policy issues. Since the first DEA-based CI model proposed by Melyn and Moesen (1991), various indexes have been developed by using the DEA technique. The environmental performance index [Färe et al., 2004], the human development index [Despotis, 2005], the macroeconomic performance index [Ramanathan, 2006], the sustainable energy index [Zhou et al., 2007], the internal market index [Cherchye et al., 2007], the technology achievement index [Cherchye et al., 2008], and the road safety performance index [Hermans, 2009], are examples among others. Furthermore, as a valuable extension of the basic DEA-based CI model, Shen et al. $(2011 ; 2012)$ proposed a generalized multiple layer DEA model (MLDEA) and a MLDEA-based CI model, which took the layered hierarchy of indicators into account.

More specifically, suppose that a set of $n$ DMUs is to be evaluated in terms of $s$ indicators (y) with a $K$ layered hierarchy, the MLDEA-based CI model can be formulated as follows [Shen et al., 2012]: 


$$
\begin{aligned}
C I_{0}=\max & \sum_{f_{1}=1}^{s} \hat{u}_{f_{1}} y_{f_{1} 0} \\
\text { s.t. } \quad & \sum_{f_{1}=1}^{s} \hat{u}_{f_{1}} y_{f_{1} j} \leq 1, \quad j=1, \cdots, n \\
& \sum_{f_{1} \in A_{f_{k}}^{(k)}} \hat{u}_{f_{1}} / \sum_{f_{1} \in A_{f_{k+1}^{(k+1)}}} \hat{u}_{f_{1}}=w_{f_{k} \in A_{f_{k+1}}^{(k+1)}}^{(k)} \in \Theta, \quad f_{k}=1, \cdots, s^{(k)}, \quad k=1, \cdots, K-1 \\
& \hat{u}_{f_{1}} \geq 0, \quad f_{1}=1, \cdots, s
\end{aligned}
$$

where $s^{(k)}$ is the number of categories in the $k^{\text {th }}$ layer $(k=1,2, \ldots, K) . s^{(l)}=s$. $A_{f_{k}}^{(k)}$ denotes the set of indicators of the $f^{\text {th }}$ category in the $k^{\text {th }}$ layer.

$w_{f_{k}}^{(k)}$ denotes the internal weights associated with the indicators of the $f^{\text {th }}$ category in the $k^{\text {th }}$

layer, which sum up to one within a particular category. $\Theta$ denotes the restrictions imposed to the corresponding internal weights.

The main idea of this model is to first aggregate the values of the indicators within a particular category of a particular layer by the weighted sum approach in which the sum of the internal weights equals to one. With respect to the final layer, the weights for all the subindexes are determined using the basic DEA-based CI approach. In general, the model (1) reflects the layered hierarchy of the indicators by specifying the weights in each category of each layer. Meanwhile, by restricting the flexibility of these weights, denoted as $\Theta$, consistency with prior knowledge and the obtainment of acceptable layer-specific weights are guaranteed, which cannot be realized in the one layer model. For the detailed deduction process of the model, we refer to Shen et al. $(2011 ; 2012)$.

\section{Indicators of driving behavior and their definition}

In general, driving behavior is the vehicle control in longitudinal and transverse direction. According to the European "Safety Handbook in Secondary Roads" [Gatti et al., 2007], the speed, acceleration, and lateral position are the three most common parameters to describe and analyze the behavior of a driver. These parameters are physical and geometrical values which can be measured or calculated.

\subsection{Speed}

The speed is the distance travelled divided by the time of travel. Basically, there are two different speeds: the speed which is only influenced by the traffic facility and the environment and the speed which is additionally influenced by traffic. To investigate the impacts of road geometry and environment a speed which is not influenced by traffic should be considered. For this purpose, the spot speed can be used which is the speed in a defined spot at a defined time.

\subsection{Acceleration}

The acceleration is defined as the speed change within a time interval. Regarding the direction of acceleration, there is a longitudinal and transverse acceleration. The longitudinal acceleration is a value of speed change and can be used, as well as the centrifugal accelera- 
tion, as comfort criterion which gives information about how fast a driver changes the speed or which curve speed is accepted.

\subsection{Lateral position}

The lateral position is the position of the vehicle within a lane. It is a geometrical value which is e.g., the distance between the center of the road and the vehicle's longitudinal axis. This indicator gives the possibility to analyze the driven track. Especially in curves the lateral position of cars is a perfect indicator to investigate corner cutting.

\section{Data collection and analysis}

This study aims to investigate the driving behavior of different drivers in and nearby a curve. Horizontal curves, particularly on two-lane rural roads, have been recognized as a significant safety issue for many years: crash rates are 1.5 to 4 times higher on horizontal curves than on straight road sections, and $25-30 \%$ of all fatal accidents occur in curves [SafetyNet, 2009a].

In doing so, 129 drivers - aged between 18 and 54 years old - were enrolled to drive, using a fixed-based high-fidelity driving simulator (STISIM M400; Systems Technology Incorporated), in an existing stretch of two-lane rural road in Belgium with a left-oriented compound curve, preceded by a long tangent and characterized by complex geometrical alignment. Data on the three aforementioned driving performance indicators, i.e., speed, acceleration and lateral position, are collected from the simulator.

\subsection{Data processing}

Data analysis for these three indicators are based on values obtained at eight different measurement points along the driving scenario, i.e., $\mathrm{P} 1=500 \mathrm{~m}, \mathrm{P} 2=166 \mathrm{~m}$ and $\mathrm{P} 3=50 \mathrm{~m}$ before curve, $\mathrm{P} 4=$ curve entry, $\mathrm{P} 5=$ middle curve, $\mathrm{P} 6=$ curve end, and $\mathrm{P} 7=50 \mathrm{~m}, \mathrm{P} 8=100 \mathrm{~m}$ after curve, for each driver. Before using the raw data in the model, the following process was conducted for each point, separately.

\subsubsection{Speed}

Apart from the emergency services, nobody should drive faster than the legal speed limit. As a result, based on their driven speed, i.e., below or equal to $70 \mathrm{~km} / \mathrm{h}$ on the one hand and above $70 \mathrm{~km} / \mathrm{h}$ on the other. Next, by using hierarchical cluster analysis in SPSS, each group is further divided into several sub-groups. Finally, all the sub-groups were assigned ascending grades starting from 1, illustrating the degree of each driver's performance, so that the lower the grade, the better the performance. This process is carried out in each of the eight points, respectively. Table 2 . shows the results of clusters at point $1(500 \mathrm{~m}$ before the curve).given the posted speed limit of the road in the simulated and real environment of $70 \mathrm{~km} / \mathrm{h}$, all the drivers are first divided into two groups.

Table 2. The threshold of speed clusters at point 1 (500m before the curve)

\begin{tabular}{llllll}
\multicolumn{2}{l}{ Drivers driving with a speed $\leq 70 \mathrm{Km} / \mathrm{h}$} & \multicolumn{4}{l}{ Drivers driving with a speed $>70 \mathrm{Km} / \mathrm{h}$} \\
\hline Speed range & Nr. of drivers (\%) & Grade & Speed range & Nr. of drivers $(\%)$ & Grade \\
$(68.54,70.00]$ & $35(27.13 \%)$ & Nr. 1 & $(70.53,77.47)$ & $43(33.33 \%)$ & Nr. 4 \\
$(64.66,68.15)$ & $19(14.73 \%)$ & Nr. 2 & $(77.58,86.07)$ & $20(15.50 \%)$ & Nr. 5 \\
$(59.03,61.59)$ & $8(6.2 \%)$ & Nr. 3 & $(96.25,106.00)$ & $4(4.65 \%)$ & Nr. 6 \\
\hline
\end{tabular}




\subsubsection{Acceleration}

Like speed, the hierarchical cluster analysis is applied on the acceleration data at different points. As a result, each group is allocated a grade indicating its performance. Table 3. shows an example of grading at point 4 (curve entry).

Table 3. The threshold of acceleration clusters at point 4 (curve entry)

\begin{tabular}{llc}
\multicolumn{2}{l}{ Threshold for “Acceleration” in point 4} \\
\hline Acceleration range & Nr. of drivers $(\%)$ & Grades \\
$(0.273,0.736)$ & $43(33.33 \%)$ & Nr. 1 \\
$(0.751,0.920)$ & $36(27.91 \%)$ & Nr. 2 \\
$(0.936,1.126)$ & $24(18.60 \%)$ & Nr. 3 \\
$(1.151,2.439)$ & $26(20.16 \%)$ & Nr. 4 \\
\hline
\end{tabular}

\subsubsection{Lateral position}

Since the road width in the simulator scenario is $2.8 \mathrm{~m}$, based on the average passenger car dimension, drivers are assigned a grade according to Table 4 . A score of 1 indicates best performance because he/she drives in almost the middle of the lane, while a score of 3 is given to the worst performers because they pass either the center-line or edge-line of the road. Finally, drivers not belonging to these two groups are assigned a score of 2 .

Table 4. The threshold of lateral position clusters

\begin{tabular}{rc}
\hline Threshold for "Lateral Position" & Grades \\
\hline $1.3 \leq \mathrm{LP} \leq 1.5$ & Nr. 1 \\
$0.95<\mathrm{LP}<1.3$ or $1.5<\mathrm{LP}<1.85$ & Nr. 2 \\
$\mathrm{LP} \leq 0.95$ or $\mathrm{LP} \geq 1.85$ & Nr. 3 \\
\hline
\end{tabular}

\subsection{Model preparation}

In this study, the MLDEA-based CI model is applied to evaluate the driving performance of each of the 129 drivers by taking into account all the 24 hierarchically structured indicators (see Fig. 1.). In doing so, the model (1) is restructured by minimizing the resulting index score $^{1}$, subject to the condition that the set of weights obtained in this manner for each driver must also be feasible for all the other drivers included in the calculation.

\footnotetext{
${ }^{1}$ Remember that the raw data have been transformed on an ordinal scale with 1 representing the best category.
} 


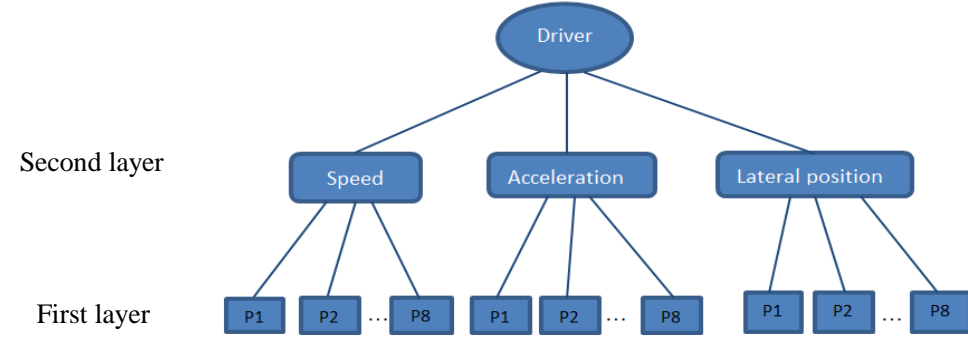

Fig. 1. Hierarchically structured driving behavior indicators

With respect to the weight restrictions, for the first layer, the driving performance during the curve is considered to be more important than before or after the curve. Therefore, a relative weight restriction is given ensuring that the indicators in and during the curve, i.e., at points P4, P5, and P6, receive a higher weight than the other points. Regarding the second layer, to guarantee that all the three indicators of driver behavior - speed, acceleration and lateral position - will be used to some extent by the model, the share of each of these three factors in the final index score is restricted to be equal with $30 \%$ variability, which thereby lies between $0.233(=1 / 3 \times 0.7)$ and $0.433(=1 / 3 \times 1.3)$, respectively, as lower and upper bound, yet is rather broad to allow a high level of flexibility.

\section{Results}

\subsection{Index scores and drivers ranking}

By the application of the MLDEA-based CI model, 24 driver performance indicators are now combined into a composite index score for each driver by selecting the best possible indicator weights under the imposed restrictions. As a result, the index score of each driver is calculated in relation to all the other drivers. In case the lowest possible index value is equal to 1 , a best performer is identified. Underperforming drivers will obtain an index value larger than 1. Apart from identifying the best-performing and underperforming drivers, it is possible to rank them based on their calculated index scores (see Table 5.).

Table 5. Drivers`ranking

\begin{tabular}{|l|c|c|c|c|l|l|l|l|l|l|c|}
\hline Driver's Nr. & 48 & 49 & 47 & 106 & $\ldots$ & 95 & 21 & 74 & 84 & 28 & 63 \\
\hline Index Score & 1 & 1 & 1 & 1.01 & $\ldots$ & 1.98 & 1.99 & 2.00 & 2.01 & 2.03 & 2.08 \\
\hline
\end{tabular}

Moreover, Table 6 shows the categorization of the drivers into five groups based on their index scores. Drivers with index score larger than 1.25, typically should receive additional training or performance review by supervisors. Furthermore, it is possible to help each driver to improve his/her performance by means of training in the aspect of the most problematic parameter.

Table 6. Drivers categories

\begin{tabular}{cccc}
\hline Performance & Index score & Nr. of drivers in each group & Percentage of drivers \\
\hline High & 1 & 2 & $1.55 \%$ \\
Relatively high & $(1,1.25]$ & 45 & $34.88 \%$ \\
\hline
\end{tabular}




\begin{tabular}{cccc}
\hline Medium & $(1.25,1.5]$ & 45 & $34.88 \%$ \\
Relatively low & $(1.5,1.75]$ & 19 & $14.74 \%$ \\
low & $>1.75$ & 18 & $13.95 \%$ \\
\hline
\end{tabular}

\subsection{Comparison of drivers in terms of driving performance indicators}

In order to make comparison between best-performing and underperforming drivers, their performance in each aspect is depicted in the following sections.

\subsubsection{Speed}

Speed is at the core of the road safety problem. Very strong relationships have been established between speed and crash risk and severity. In fact, speed is involved in all accidents: no speed, no accident. In around $30 \%$ of the fatal accidents, speed is an essential contributory factor [SafetyNet, 2009b]. At a higher speed, it is more difficult to react in time and prevent an accident. Fig. 2 shows the speed of best-performer versus two worst-performers. The two underperforming drivers are distinguished as worst-performer either because of their high speed or evasive changes during curve. The best-performer, on the contrary, drives smoothly bellow posted speed limit.

Fig. 2. The speed of the best-performer versus two worst-performing drivers

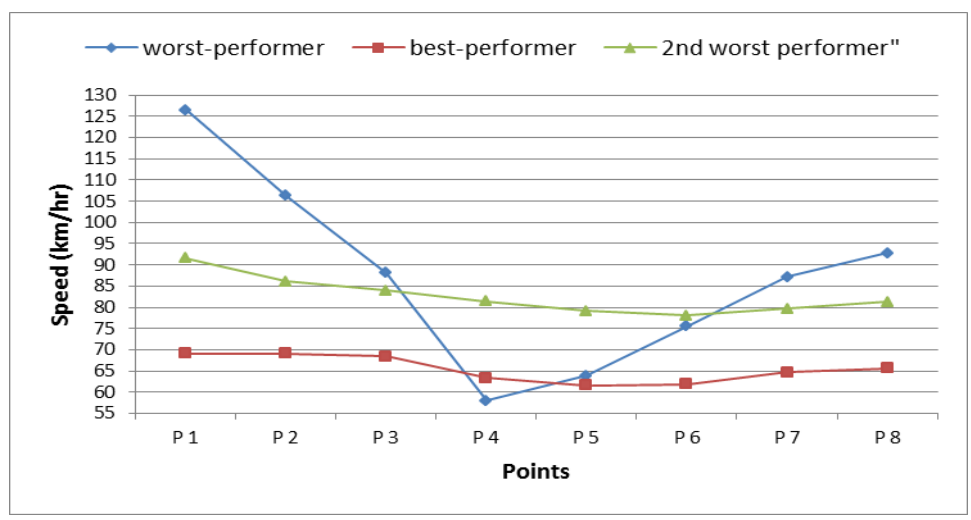

\subsubsection{Acceleration}

The total acceleration can be decomposed into longitudinal acceleration and lateral acceleration. The longitudinal acceleration, indicating how fast a driver changes her/his speed, is shown in the left-hand side of Fig. 3. According to Lamm and Chouriri (1987), the observed deceleration rates when approaching horizontal curves should not be significantly different from $-0.85 \mathrm{~m} / \mathrm{s}^{2}$. Even though others proposed higher acceptable values up to $1.34 \mathrm{~m} / \mathrm{s}^{2}$ and $-1.8 \mathrm{~m} / \mathrm{s}^{2}$ [Hu and Donnel, 2010]. It can be seen that the worst-performer exceeded dramatically the maximum threshold when approaching and leaving the curve. On the other hand, the lateral acceleration - indicative of how fast a driver changes her/his di- 
rection, shown in the right-hand side of Fig. 3. - confirms inappropriate driving behavior of the worst performer.

Fig. 3. The acceleration of the best-performer versus the worst-performer

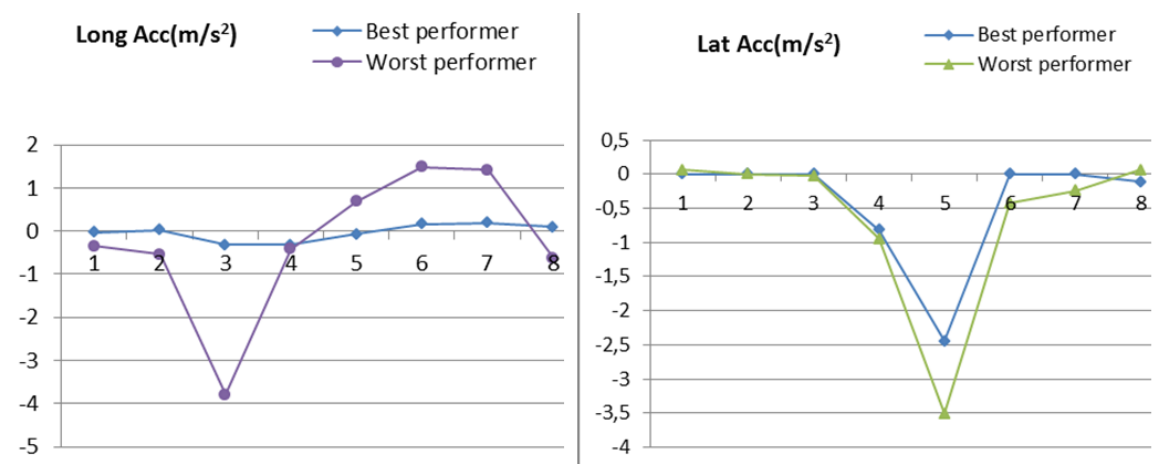

\subsubsection{Lateral position}

When driving, it is commonly accepted that the higher the variability in the lateral position of a vehicle, the less safe of a driver [COST, 1999]. By comparing the performance of the best-performer and the worst-performer with respect to their lateral positions in this experiment shown in Fig. 4., it is easy to see that the worst performer was involved in more dangerous situation. However, according to the threshold of lateral position indicated in Table 4., it should be noted that although the best-performer in this experiment was doing obviously better than the worst one, he was still not doing perfect, especially when he was entering and leaving the curve.

Fig. 4. The lateral position of the best-performer versus the worst-performer

Lateral Position(m)

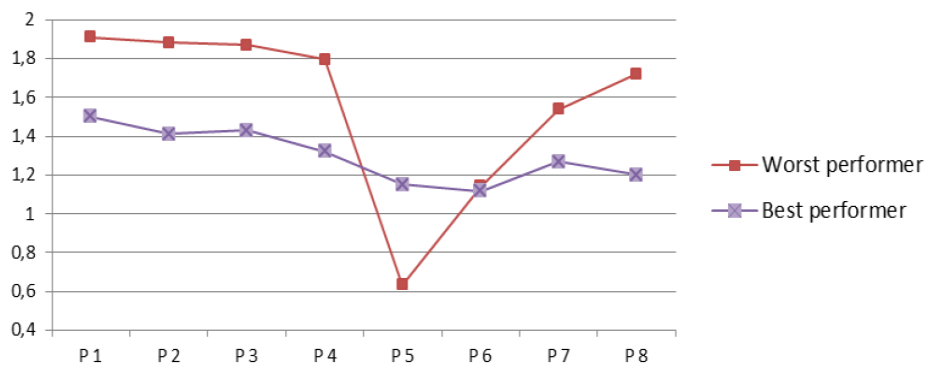

\section{Sensitivity analysis}

It is important to rigorously investigate the robustness of the indexes or the stability in the output (i.e., drivers' ranking) given small changes in the indicator set. Hence, we considered following 8 scenarios: in scenario 1 , the point 1 (500 $\mathrm{m}$ before curve) is excluded; in 
scenario 2, the point 2 (166 m before curve) is excluded, and so on. In each scenario, the index score of each driver was recalculated. The results for the 10-first and 10-last drivers in the ranking are presented in Fig. 5., which provide insight into the sensitivity of each driver's scores with respect to each scenario and indicate driver nr. 48 as a robust, overall best performing driver who obtains an index score of one in all the scenarios.

Fig. 5. Boxplot of drivers when eliminating indicators at one point at a time (10-first and 10-last drivers in the ranking)

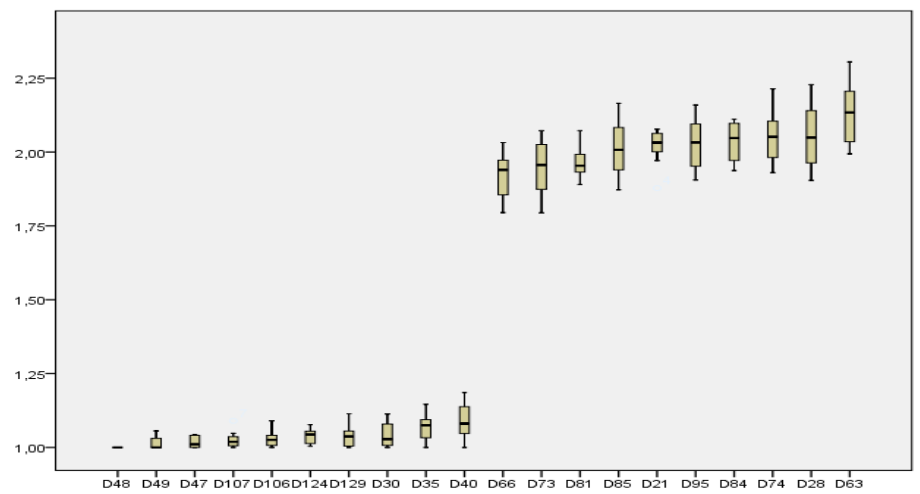

\section{Conclusion and future research}

In order to measure the multi-dimensional concept of driving performance which cannot be captured by a single indicator at one point in time, we investigated in this study the construction of a composite driving performance index for drivers evaluation. In doing so, a multiple layer DEA-based composite index model was applied on a hierarchy of driving performance indicators. Based on this model, the most optimal driving performance index score for each of the 129 drivers was determined by combining all the 24 hierarchical indicators. Apart from identifying the best-performing and underperforming drivers, all drivers were ranked based on their calculated index scores, and their relative performance with respect to speed, acceleration, and lateral position was compared. In addition, robustness of the results was checked by means of sensitivity analysis. Based on the results, it would be useful to train particular drivers in different tasks in the simulator, according to each driver's weakness.

Future research on the composite driving performance index can be done concerning the data, i.e. adjusting the model in order to allow the use of raw data instead of assigned grades to different indicator clusters. In addition, other road types or other sections of road (e.g., intersections) as well as roads with different speed limits may be considered.

\section{References}

Charnes A., Cooper W. W. and Rhodes E. Measuring the efficiency of decision making units. European Journal of Operational Research, 2(1978) 429-444.

Cherchye, L., Moesen, W., Rogge, N., and van Puyenbroeck, T., Saisana. An introduction to "benefit of the doubt" composite indicators. Social Indicators Research, 82(2007) 111-145. 
Cherchye, L., Moesen, W., Rogge, N., Van Puyenbroeck, T., Saisana, M., Saltelli, A., Liska, R., and Tarantola, S. Creating composite indicators with DEA and robustness analysis: The case of technology achievement index. Journal of the Operational Research Society, 59(2008) 239-251.

Cooper, W.W., Seiford, L.M. and Tone, K. Data envelopment analysis: A Comprehensive text with models, applications, references and DEA-solver software, Kluwer Academic Publishers, Dordrecht (2000).

Despotis, D.K. A reassessment of the human development index via data envelopment analysis, Journal of the Operational Research Society, 56(8) (2005) 969-980.

ERF European road statistic (2012), www.erf.be

European road safety observatory (2006), Retrived on March 5, 2007 from www.erso.eu

Färe, R., Grosskopf, S., and Hernandez-Sancho, F. Environmental performance: An index number approach. Resource and Energy Economics, 26 (2004) 343-352.

Gatti, G., Polidori (Poliba), C., Galvez, I., Mallschutzke (Ineco), K., Jorna, R., Van de Leur (Mobycon), M., Dietze, M., Ebersbach, D., Lippold, C., Schlag, B., Weller (Tud), G., Wyczynski (Piap), A., Iman, F., Aydin (TGDH), C. Safety Handbook for Secondary Roads, Final Report (2007) Contract No. 508164, RIPCORD-ISEREST Deliverable D13.

Golany, B., Roll, Y. An application procedure for DEA. Omega-The International Journal of Management 17 (1989) 237-250.

Hermans, E. A methodology for developing a composite road safety performance index for crosscountry comparison, (2009) PhD thesis, Hasselt university, Hasselt, Belgium.

$\mathrm{Hu}, \mathrm{W}$., and Donnel, E.T. Models of acceleration and deceleration rates on a complex two-lane rural highway: Result from a nighttime driving experiment. Transportation Research, part F, 13(6) (2010) 397- 408 .

Lamm, R., and Choueiri, E.M. A design procedure to determine critical dissimilarities in horizontal alignment and enhance traffic safety by appropriate low-cost or high-cost projects, (1987) National Science Foundation, Washington.

Melyn, W. and Moesen, W. Towards a synthetic indicator of macroeconomic performance: Unequal weighting when limited information is available, Public Economics Research, paper 17(1991) CES, KU Leuven.

Pedraja-Chap arro F., Salinas-Jimenez J., and Smith P. On the role of weight restrictions in data envelopment analysis. Journal of Productivity Analysis 8 (1997) 215-230.

Ramanthan, R. Evaluating the comparative performance of countries of Middle East and North Africa: A DEA application, Socio-Economic Planning Sciences, 40 (2006) 156-167.

SafetyNet (2009a) Roads, Retrieved on July 12, 2011.

SafetyNet (2009b) Speeding, Retrieved on July 15, 2010

Saisana M., Saltelli A., and Tarantola S. Uncertainty and sensitivity analysis as tools for the quality assessment of composite indicators. Journal of the Royal Statistical Society series A, 168 (2005) 1-17 Thanassoulis, E., Portela, M. C. and Allen, R. Incorporating value judgments in DEA, in Cooper, W. W., Seiford, L., and Zhu, J. (eds), Handbook on data envelopment analysis, Kluwer Academic Publishers, Dordrecht, (2004) 99-138.

Srinivasan, R., Beak, J., Carter, D., Persaud, B., Lyon, C., Eccles, K., Gross, F., and Lefler, N. Safety evaluation of improved curve delineation, (2009) Publication FHWA-HRT-09-045, US Department of Transportation.

Shen, Y., Hermans, E., Brijs, T. and Wets, G. Data envelopment analysis for composite indicators: A multiple layer model. Social Indicators Research. (2012) DOI: 10.1007/s11205-012-0171-0.

Shen, Y., Hermans, E., Ruan, D., Wets, G., Brijs, T., and Vanhoof, K. A generalized multiple layer data envelopment analysis model for hierarchical structure assessment: A case study in road safety performance evaluation. Expert System with Applications, 38(12) (2011) 15262-15272.

Treat, J. R., Tumbas, N. S., McDonald, S. T., Shinar, D., Hume, R.D., Mayer, R.E., Stanisfer, R.L. and Castellan, N.J. Tri-level study of the causes of traffic accidents, report No. DOT-HS-034-3-535-77 (TAC) (1977).

Zhou, P., Ang, B.W., and Poh, K.L. A mathematical programming approach to constructing composite indicators. Journal of the Ecological Economics, 62 (2007) 291-297. 